Research Paper

\title{
Enhancing photon collection from quantum emitters in diamond
}

\author{
Michael TRUPKE ${ }^{1}$, William J. MUNRO ${ }^{2}$, Kae NEMOTO ${ }^{3}$, and Jörg SCHMIEDMAYER ${ }^{4}$ \\ 1,4 Vienna Center for Quantum Science and Technology \\ ${ }^{2}$ NTT Basic Research Laboratories \\ ${ }^{3}$ National Institute of Informatics
}

\section{ABSTRACT}

Diamond has recently become one of the leading candidates for applications in quantum communication and quantum computing. Diamond color centers are ideal as single photon sources. In this article we give an overview of the various techniques that can be used to improve the collection of photons from these emitters. These range from solid immersion lenses to Purcell-enhancement in microstructures and microcavities.

\section{KEYWORDS}

Quantum Emitters, Quantum Communication, Diamond color centers

\section{Introduction}

In recent years, diamond has advanced from being simply a valuable gemstone to one of the most fervently studied technical materials [1]. Diamond is taking on the role as a practical and versatile platform for harnessing the power of quantum physics, This is largely due to the fact that high-purity artificial diamond, which retains the most important technical properties of the naturally-occurring variant, can now be routinely manufactured. Diamond is the hardest known material, and diamond nanocrystals are now routinely applied to tool surfaces to improve their durability [2]. Diamond presents extremely high thermal conductivity, exceeding $2 \mathrm{~kW} /(\mathrm{m} \cdot \mathrm{K})$, and has therefore found use in highpower electronics. Diamond is also a leading contender as the material of choice for the quantum computer industry both as a source of matter and optical qubits.

Researchers have begun to study in detail the optical properties of color centers hosted in diamond. Pure diamond has a vast transparency band ranging from the ultraviolet $(220 \mathrm{~nm})$ through the optical regime to the microwave regime. Impurities in diamond lead to coloration at various wavelengths. Over 500 luminescent diamond defects [3] have so far been documented, and a number of these have properties which render them

Received December 31, 2010; Accepted January 6, 2011.

${ }^{1)}$ mtrupke@ati.ac.at, ${ }^{2)}$ bilmun@qisl.ex.nii.ac.jp,

4) schmiedmayer@atomchip.org

DOI: 10.2201/NiiPi.2011.8.4 candidates of interest as high-efficiency single-photon sources, luminescent markers for biological imaging [4] and stationary qubits for quantum information processing. The most common impurity in diamond is nitrogen, and this forms the best-studied color center, the Nitrogen-Vacancy center which we depict in Fig. 1. These centers occurs in two variants, one neutral $\left(\mathrm{NV}^{0}\right)$ and one negatively-charged $\left(\mathrm{NV}^{-}\right)$.

It is this latter center that has been the subject of intense study as it possesses a multitude of properties of interest: It has a spin-triplet in its electronic ground

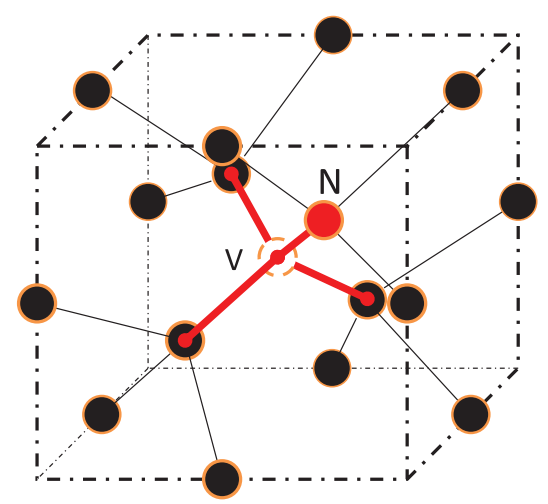

Fig. 1 Schematic representation of a Nitrogen-Vacancy center in Diamond. 
Table 1 Properties of various diamond centers. Here $\lambda$ is the wavelength of the zero-phonon line, $\Delta \lambda$ is the typical spectral width, $\gamma_{t o t}$ is the excited-state amplitude decay rate, and $\eta_{\text {tot }}$ is the branching ratio of the transition compared to all other decay channels. The nickel-nitrogen center (NE8, [17]) and the silicon-vacancy (SiV, [20], [21]) are naturally-occurring impurities, while the chromium [18], [19] and nickel-silicon center [22] were created by implantation.

\begin{tabular}{|c|c|c|c|c|c|c|}
\hline & $\begin{array}{c}\lambda \\
\mathrm{nm}\end{array}$ & $\begin{array}{l}\Delta \lambda \\
\mathrm{nm}\end{array}$ & $\begin{array}{c}\sigma_{E} \\
\mu \mathrm{m}^{2}\end{array}$ & $\begin{array}{c}\gamma_{t o t} \\
\mathrm{MHz} / 2 \pi\end{array}$ & $\eta_{t o t}$ & $\begin{array}{c}\text { Ground } \\
\text { states }\end{array}$ \\
\hline $\mathrm{NV}$ & 637 & 200 & 0.19 & 6.9 & 0.04 & $0, \pm$ \\
\hline $\mathrm{SiV}$ & 738 & 7 & 0.26 & 29.5 & 0.05 & multiple \\
\hline NE8 & 800 & 1.2 & 0.30 & 6.9 & 0.7 & not known \\
\hline $\mathrm{Cr}$ & 749 & 4 & 0.27 & 80 & 0.3 & not known \\
\hline $\mathrm{NiSi}$ & 770 & 5 & 0.28 & 40 & $\sim 1$ & not known \\
\hline
\end{tabular}

state which possesses ultra-long coherence time at room temperature - on the order of $2 \mathrm{~ms}$ in isotopicallypurified diamond [5]. It fluoresces brightly with nearunit quantum efficiency, in a wavelength range between $600 \mathrm{~nm}$ and $800 \mathrm{~nm}$ which is convenient for high-efficiency detection using standard silicon-based avalanche photodiodes. Its zero-phonon line lies at $637 \mathrm{~nm}$, and is therefore accessible with low-cost solidstate diode lasers. Next, the rate of fluorescence is spin-dependent and so the qubit state can be read out optically. $\mathrm{NV}^{-}$is therefore ideally suited as a unit for room-temperature quantum information processing and storage. Even with all these advantages the centre is not the best candidate for the development of narrowband source of single photons for long-distance communication due to its short wavelength and broadband emission range. Diamond however hosts a large number of other emitters many with more favorable properties. The main properties of some of the more prominent emitters are listed in the table below. One of the other key issues that needs to be considered is the collection efficiency from the emitters and how it can be improved.

In this article we will describe and provide an overview of the various techniques used to improve the collection of photons from diamond emitters. These techniques range from solid immersion lenses to Purcell-enhancement in microstructures and microcavities.

\section{Collection enhancement of photons from diamond emitters}

We will now describe the various techniques that can be used to enhance the collection of photons from the diamond emitters. First however we need to describe how photons are generally collected. In a standard fluorescence collection setup, the collection efficiency is limited by the numerical aperture (NA) of the collection objective. The effective numerical aperture of the collection optics is however reduced by refraction of rays exiting the plane diamond surface, to $\mathrm{NA}(\mathrm{eff})=\mathrm{NA} / n_{D}$, where $n_{D}=2.4$ is the refractive index of diamond. Total internal reflection at the surface will lead to a further reduction in the count rate. To counter these effects, a variety of techniques have been developed to improve the collection efficiency for light emitted by single color centers. For experiments with $\mathrm{NV}^{-}$, the motivation is to improve the rate and fidelity of the qubit state readout. The aim using other emitters is to create high-efficiency single photon sources, for example for applications in quantum cryptography and metrology.

Conceptually, the simplest approach is given by solid-immersion lensing. The improvement is given firstly by the fact that total internal reflection can be drastically reduced if the diamond surface is shaped such that photons impinge upon it at near-normal incidence, i.e. if the surface of the diamond matches the shape of the wavefront of the emission pattern. For a radiating dipole, this can be achieved to a very good approximation by creating a spherical surface with the emitter at its center. In this geometry, photons exiting the diamond will furthermore not be refracted. The team of Jelezko and Wrachtrup [7] recently demonstrated the efficacy of this approach having increased the photon count rate from a single $\mathrm{NV}^{-}$center by almost an order of magnitude.

More sophisticated approaches use micro-fabricated structures to tailor the properties of the electromagnetic field modes which couple to the emitter. This in turn affects the rate and pattern of the emission. Three main strategies have so far been applied to achieve enhancement of this type:

- Dielectric nano-antennae,

- Plasmonics and

- Microresonators.

Babinec et al. [8] has fabricated nanowires in diamond of $200 \mathrm{~nm}$ diameter and $2 \mu \mathrm{m}$ length, some of which contained single $\mathrm{NV}^{-}$centers. In this configuration, the dipole radiation pattern is enhanced in the direction of the axis of the nanowire by its small mode volume leading to an order of magnitude enhancement in the collected photon count rate compared to emitters in bulk diamond. Similar enhancements were achieved in the group of Benson [9] using plasmonic enhancement from gold microspheres which were brought close to a diamond nanocrystal. Both of these techniques, 
while offering an enhancement of the emission rate, provide almost no spectral selectivity. To this end, both spatial and spectral properties of the surrounding electromagnetic field need to be modified, which can be achieved with microresonators. A successful example of this concept was presented by Englund et al. [10], who demonstrated coupling to a photonic crystal cavity with a $Q$ of 610 , fabricated in gallium phosphide. In their experiment, the count rate from a single $\mathrm{NV}^{-}$center in a bulk diamond sample was increased by a factor 7 selectively within the resonance of the photonics crystal cavity, which had a half-width of $\kappa=2 \pi \times 768 \mathrm{GHz}$. A weak second enhancement peak was detected which coincided with a further resonance of the cavity. Other efforts in this direction include coupling of nanodiamonds to wavelength-scale, low-finesse Fabry-Perot resonators [11] and to high-Q microdisk resonators [12].

\section{Enhancement with fiber-coupled mi- crocavities}

In our work, we are using emitters in diamond to create high-efficiency single-photon sources [13]. A schematic of our envisaged device is shown in Fig. 2 and is composed from the top down of a fiber/wave guide coupled to a dielectric mirror. Next to this mirror is a diamond layer with the emitter embedded in it followed by an anti-reflection layer. Finally comes an actuated silicon mirror. The proposed design is scalable as it relies on well-proven silicon microfabrication techniques and standard fiber-optics components. Similar designs using Rubidium atoms [14] (instead of diamond centers with emitters) have shown enhancement of fluorescence and single atom detection sensitivity. In more detail the microcavities consist of a microfabricated concave mirror etched into a silicon wafer, and a

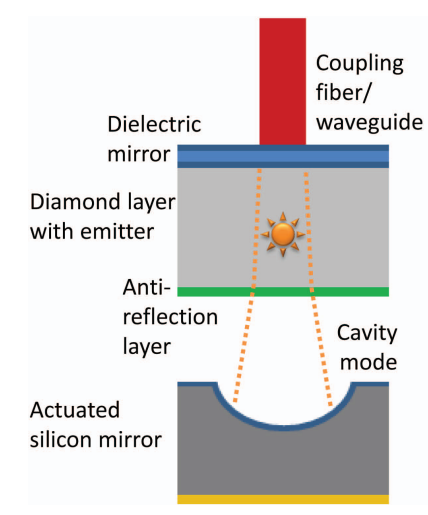

Fig. 2 Schematic of a fiber-coupled resonator containing a single emitter in a diamond layer. plane mirror formed at the end of a single-mode optical fiber or waveguide. The construction enables the creation of small mode waists and high finesse, allowing high potential enhancement factors. An advantage of this design is that the mirrors on the silicon surface can be created in large arrays, and are intrinsically compatible with other standard fabrication procedures for silicon which may enable the integration of electronic and electromechanical elements. Furthermore, the mode size of the resonator can be adapted by an appropriate choice of length and mirror radius of curvature to match the waist of a single-mode fiber. Thereby photons created inside the resonator are coupled directly into the fiber mode.

Now in the limit of high efficiency, the total conversion efficiency from an electronic excitation of the emitter to a photon exiting the resonator into the fiber mirror is given by [15]

$$
\eta_{o p t} \simeq 1-\sqrt{\frac{1}{2} \frac{\ell_{t o t}}{\eta_{t o t}} \frac{f_{L}}{n_{D}} \frac{\sigma_{C}}{\sigma_{E}}} .
$$

where $\sigma_{E}=3 \lambda^{2} / 2 \pi$ is the emitter scattering crosssection and $\sigma_{C}=\pi w_{C}^{2}$ the cavity mode area. All undesired loss channels are collected in $\ell_{\text {tot }}=\ell_{1}+T_{2}+\ell_{2}$. For a cavity with mixed refractive indices, the mode volume is modified by the refractive index of the diamond $\left(n_{D}\right)$, and this is taken into account with the factor $f_{L}=\left(L_{v a c}+n_{D} L_{D}\right) /\left(L_{v a c}+n_{D}^{2} L_{D}\right)[6]$. The expression (1) is pessimistic in nature, but is generally accurate to within $2 \%$ for $\eta_{\text {opt }}>80 \%$. Generally it is advantageous to combine a large radiative branching ratio, weak non-radiative transitions, low losses, a small mode waist and a large wavelength. The latter is also beneficial as roughness losses at the mirror surfaces and interfaces decrease for longer wavelengths. Furthermore, propagation through long-distance optical fiber links is limited by Rayleigh scattering which leads to losses proportional to $1 / \lambda^{4}$.

On the silicon mirror, scattering losses due to surface roughness $\ell_{\sigma M}$ are given by

$$
\ell_{\sigma M}=1-e^{-\left(\frac{4 \pi \sigma_{M}}{\lambda}\right)^{2}}
$$

where $\sigma_{M}$ is the RMS-roughness of the surface of the mirror substrate. In addition to the losses caused by roughness on the silicon mirror surface, the imperfections of the diamond layer will cause additional scattering. The losses $\ell_{\sigma I}$ due to the passage of a beam through a surface with roughness $\sigma_{I} \ll \lambda$, in a single pass, and losses $\ell_{\sigma D M}$ for reflection from the mirror on the external surface of the diamond, are given by

$$
\ell_{\sigma I}=1-e^{-\left(\left(n_{D}-1\right) \frac{2 \pi \sigma_{I}}{\lambda}\right)^{2}},
$$




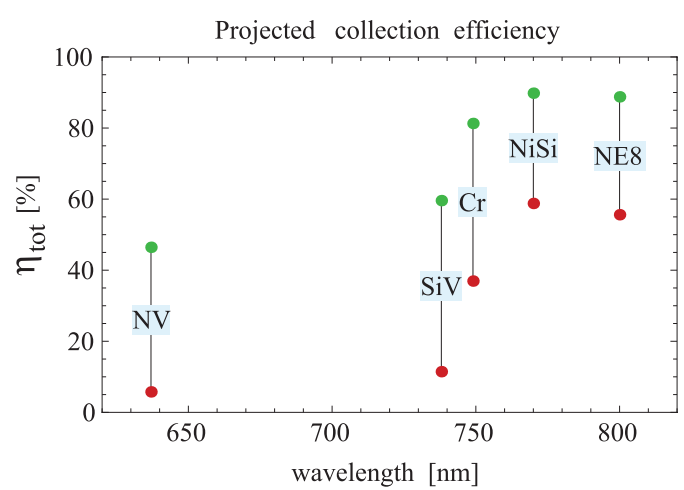

Fig. $3 \eta_{o p t}$ for a selection of diamond emitters, assuming losses between $0.2 \mathrm{~nm}$ (green points) and $1 \mathrm{~nm}$ (red points) roughness on all surfaces. Further it is assumed that the microcavity has a beam waist of $2.5 \mu \mathrm{m}$, and consists of a diamond layer of $20 \mu \mathrm{m}$ and a vacuum gap of $50 \mu \mathrm{m}$.

$$
\ell_{\sigma D M}=1-e^{-\left(\frac{4 \pi \sigma_{I}}{\lambda / n_{D}}\right)^{2}} .
$$

Currently, the best commercial CVD diamond plates have a specified roughness of $\sigma_{D} \sim 5 \mathrm{~nm}$ RA, corresponding to $7 \mathrm{~nm}$ RMS [16]. However we have already measured roughness values under $1 \mathrm{~nm}$ on untreated commercial samples, and it should be possible to further reduce this to less than $0.2 \mathrm{~nm}$. A number of methods are currently being tested at Atominstitut, TU-Wien.

From the emitter properties and projected cavity parameters, we can calculate collection efficiency values. Figure 3 shows the result of exact calculations of this quantity for the emitters listed in table I. As an example for comparison, a SiV-center will in general only decay with $5 \%$ probability via the zero-phonon line transition, while the rest of the excited-state population is lost mainly to non-radiative transitions, as well as phonon-sideband decay channels. Of this, a standard microscope objective setup would collect on the order of $2 \%$, resulting in a collection efficiency of $\sim 0.1 \%$. A resonator with losses due to $1 \mathrm{~nm}$ RMS roughness on all surfaces, corresponding to $290 \mathrm{ppm}$, would already improve this value by over two orders of magnitude to $11.4 \%$, and in principle this can be collected with nearunit efficiency with a single-mode fiber.

\section{Discussion}

As we have mentioned above, diamond color centers are ideal emitters of photons for use in quantum key distribution and quantum communication and computation applications. In all these applications an important consideration is the collection efficiency of the photons from the emitters. Especially for computa- tional purposes we need this as close to unity as possible. To maximize this efficiency and get it as close to unity we need the following: a large radiative branching ratio, weak non-radiative transitions, low losses, a small mode waist and a large wavelength. Many of these properties are a function of the color center but a high-finesse fiber-coupled Fabry-Perot resonator significantly helps. Still even in the best of cases we considered this efficiency is likely to be below ninety percent which will have significant implications for quantum gates and quantum computation.

\section{Acknowledgments}

This work was supported in part by the Austrian Nano-initiative PLATON NAP, the Wittgenstein prize, the European Union seventh framework projects HIP, and the Japanese Funding Program for World-Leading Innovative R\&D on Science and Technology (FIRST).

\section{References}

[1] S. E. Coe and R. S. Sussmann, "Optical, thermal and mechanical properties of CVD diamond," Diamond Rel. Mat., vol.9, p.1726, 2000.

[2] B. Lux and R. Haubner, "Diamond deposition on cutting tools," Ceramics International, vol.22, no.4, p.347, 1996.

[3] A. M. Zaitsev, Optical Properties of Diamond: A Data Handbook, Springer, 2001.

[4] F. Neugart, A. Zappe, F. Jelezko, C. Tietz, J. P. Boudou, A. Krueger, and J. Wrachtrup, "Dynamics of Diamond Nanoparticles in Solution and Cells," Nano Lett., vol.7, p.35883591, 2007.

[5] G. Balasubramanian, P. Neumann, D. Twitchen, M. Markham, R. Kolesov, N. Mizuochi, J. Isoya, J. Achard, J. Beck, J. Tissler, V. Jacques, P. R. Hemmer, F. Jelezko and J. Wrachtrup, "Ultralong spin coherence time in isotopically engineered diamond," Nature Materials, vol.8, p.383, 2009.

[6] J. Gerard and B. Gayral, "Strong Purcell Effect for InAs Quantum Boxes in Three-Dimensional Solid-State Microcavities," J. Lightwave Techn., vol.17, no.11, p.2089, 1999.

[7] P. Siyushev, F. Kaiser, V. Jacques, I. Gerhardt, S. Bischof, H. Fedder, J. Dodson, M. Markham, D. Twitchen, F. Jelezko and J. Wrachtrup, "Integrated Diamond Optics for Single Photon Detection," arXiv:1009.0607v1, 2010.

[8] T. M. Babinec, B. J. M. Hausmann, M. Khan, Y. Zhang, J. R. Maze, P. R. Hemmer and M. Lončar, "A diamond nanowire single-photon source," Nature Nanotechnology, vol.5, p.195, 2010.

[9] S. Schietinger, M. Barth, T. Aichele and O. Benson, "Plasmon-Enhanced Single Photon Emission from a Nanoassembled Metal-Diamond Hybrid Structure at 
Room Temperature," Nano Lett., vol.9, no.4, p.1694, 2009.

[10] D. Englund, B. Shields, K. Rivoire, F. Hatami, J. Vučkovic, H. Park and M. D. Lukin, "Deterministic Coupling of a Single Nitrogen Vacancy Center to a Photonic Crystal Cavity," arXiv:1005.2204, 2010.

[11] Y. Dumeige, R. Alleaume, P. Grangier, F. Treussart and J.-F. Roch, "Controlling single diamond NV color center photoluminescence spectrum with a Fabry-Perot microcavity," arXiv:1010.0779v1, 2010.

[12] P. E. Barclay, C. Santori, K.-M. Fu, R. G. Beausoleil and O. Painter, "Coherent interference effects in a nanoassembled diamond NV center cavity-QED system," Optics Express, vol.17, no.10, p.8081, 2009.

[13] C. K. Law and H. J. Kimble, "Deterministic generation of single photons," J. Mod. Opt., vol.44, p.2067, 1997.

[14] M. Trupke, J. Goldwin, B. Darquie, G. Dutier, S. Eriksson, J. P. A. Ashmore and E. A. Hinds, "Atom detection and photon production in a scalable, open, Fabry-Perot microcavity," Phys. Rev. Lett., vol.99, p.063601, 2007.

[15] M. Trupke et al., in preparation, 2010.

[16] http://www.e6cvd.com/

[17] T. Gaebel, I. Popa, A. Gruber, M. Domhan, F. Jelezko and J. Wrachtrup, "Stable single-photon source in the near infrared," N. J. Phys., vol.6, p.98, 2004.

[18] I. Aharonovich, S. Castelletto, B. C. Johnson, J. C. McCallum, D. A. Simpson, A. D. Greentree, and S. Prawer, "Chromium single-photon emitters in diamond fabricated by ion implantation," Phys. Rev. B, vol.81, p.121201(R), 2010.

[19] I. Aharonovich, S. Castelletto, B. C. Gibson, B. C. Johnson, and S. Prawer, "Imaging and quantum efficiency measurement of chromium emitters in diamond," arXiv:1008.2812, 2010.

[20] I. I. Vlasov, A. S. Barnard, V. G. Ralchenko, O. I. Lebedev, M. V. Kanzyuba, A. V. Saveliev, V. I. Konov, and E. Goovaerts, "Nanodiamond Photoemitters Based on Strong Narrow-Band Luminescence from SiliconVacancy Defects," Adv. Mater., vol.21, p.808, 2009.

[21] H. Sternschulte, K. Thonke, J. Gerster, W. Limmer, R. Sauer, J. Spitzer, P. C. Munzinger, "Uniaxial stress and Zeeman splitting of the $1.681 \mathrm{eV}$ optical center in a homoepitaxial CVD diamond film," Diamond Rel. Mat., vol.4, p.1189, 1995.

[22] I. Aharonovich, C. Zhou, A. Stacey, J. Orwa, S. Castelletto, D. Simpson, A. D. Greentree, F. Treussart, J.-F. Roch, and S. Prawer, "Enhanced single-photon emission in the near infrared from a diamond color center," Phys. Rev. B, vol.79, p.235316, 2009.

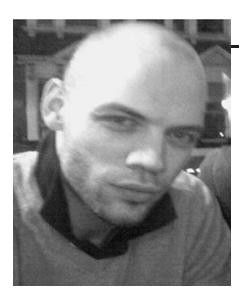

Michael TRUPKE

Michael TRUPKE is a research scientist at the Vienna Center for Quantum Science and Technology in the Institute for Atomic and Subatomic Physics at the Technical University of Vienna. His research is focused on creating light-matter interfaces for experimental quantum optics with solid state systems and ultracold atoms.

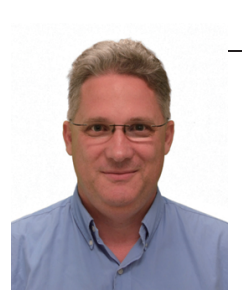

William J. MUNRO

William J. MUNRO is a research scientist within NTT Basic Research Laboratories in Japan. His current interests focus around practical implementations for optical and solid- state quantum hardware, generation of optical nonlinearities, characterization of quantum states and processes, novel quantum-communication protocols and quantum metrology.

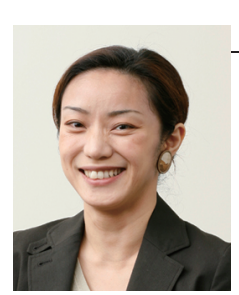

\section{Kae NEMOTO}

Kae NEMOTO is a Professor in the Principles of Informatics Research Division at the National Institute of Informatics, Tokyo, Japan. She heads the Quantum Information Science Theory Group and is a leader in the Japanese-France Laboratory for Informatics. Her research interests and efforts are currently focused around the implementation of quantum devices, architectures for large-scale QIP, quantum/atom optics and quantum nonlinear dynamics.

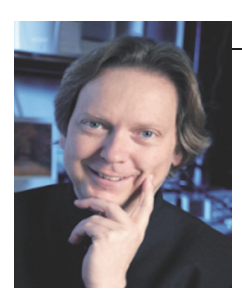

\section{Jörg SCHMIEDMAYER}

Jörg SCHMIEDMAYER is director of the Institute for Atomic and Subatomic Physics at the Technical University of Vienna and founding member of the Vienna Center for Quantum Science and Technology. His research activities include experimental quantum optics in solid state and atomic systems, microtraps for neutral atoms and atom interferometers. 